\title{
Neurexin-Neuroligin Synaptic Complex Regulates Schizophrenia-Related DISC1/Kal-7/Rac1 "Signalosome"
}

\author{
Sylwia Owczarek, ${ }^{1,2}$ Marie Louise Bang, ${ }^{1}$ and Vladimir Berezin ${ }^{1}$ \\ ${ }^{1}$ Laboratory of Neural Plasticity, Department of Neuroscience and Pharmacology, University of Copenhagen, \\ 2200 Copenhagen, Denmark \\ ${ }^{2}$ Research Laboratory for Stereology and Neuroscience, Bispebjerg Hospital, 2400 Copenhagen, Denmark
}

Correspondence should be addressed to Sylwia Owczarek; sylwia@sund.ku.dk

Received 8 January 2015; Accepted 4 May 2015

Academic Editor: Zygmunt Galdzicki

\begin{abstract}
Copyright (C) 2015 Sylwia Owczarek et al. This is an open access article distributed under the Creative Commons Attribution License, which permits unrestricted use, distribution, and reproduction in any medium, provided the original work is properly cited.

Neurexins (NXs) and neuroligins (NLs) are cell adhesion molecules that are localized at opposite sites of synaptic membranes. They interact with each other to promote the assembly, maintenance, and function of synapses in the central nervous system. Both NX and NL are cleaved from a membrane-attached intracellular domain in an activity-dependent manner, generating the soluble ectodomain of NX or NL. Expression of the NX1 and NX3 genes in the brain appears to be regulated by a schizophrenia-related protein, DISC1. Here, we show that soluble ecto-NX1 $\beta$ can regulate the expression of DISC1 and induce signaling downstream of DISC1. We also show that NL1 binds to a well-characterized DISC1 interaction partner, Kal-7, and this interaction can be compromised by DISC1. Our results indicate that the NX/NL synaptic complex is intrinsically involved in the regulation of DISC1 function, thus contributing to a better understanding of the pathology of schizophrenia.
\end{abstract}

\section{Introduction}

The development and maintenance of synaptic connections are a dynamic process that requires bidirectional interactions between pre- and postsynaptic components. Adhesion molecules are present at the synapse and organize synaptic specializations. The synaptic adhesion complex neurexin/neuroligin (NX/NL) mediates synapse formation and triggers pre- and postsynaptic signal transduction by recruiting components to developing synapses [1]. Depending on the composition of NX/NL complexes, they function as molecular switches that regulate excitatory-inhibitory (E/I) balance in the brain [1]. Mammals have three NX genes, each with two promoters. Each gene produces both longer $\alpha$-NX and shorter $\beta$-NX transcripts. Additionally, each isoform can be alternatively spliced at five and two positions, respectively [1]. Humans express five $N L$ genes, whereas other mammals express only three or four [1]. Each $N L$ gene has a canonical splicing site (SS\#A), and NL1 additionally has the SS\#B splicing site. Interestingly, the expression and function of both NXs and NLs are regulated by neural activity. $N X$ mRNA expression increases as synaptic activity increases [2], and both NX and NL can be cleaved from the pre- or postsynaptic membrane in an activitydependent manner [3-6]. The expression of the NX1 and NX3 genes was recently shown to be dysregulated in the Disc1 (disrupted in schizophrenia 1) mouse model of schizophrenia [7]. DISC1 is a scaffolding protein that plays several roles during many aspects of neural development [8]. For example, the inactivation of DISC1 during early development results in alterations in the density and morphology of dendritic spines [9]. Spines appear to be regulated by DISC1 in association with kalirin-7 (Kal-7) [10-12]. Kal-7 is an activator of a small guanosine triphosphatase (GTP), Racl. DISC1, together with Kal-7 and Racl, forms a multifunctional protein complex, called the DISC1/Kal-7/Racl signalosome, that is important for development of the nervous system [10]. Under baseline conditions, DISC1 binds Kal-7, blocking the access of Kal7 to Racl. The activation of ionotropic glutamate receptors ( $N$-methyl-D-aspartate [NMDA] receptors) weakens this protein-protein interaction, allowing free access of Kal-7 to Racl, which leads to the activation of Racl and eventually 
spine maturation [10]. The aforementioned findings led us to hypothesize that NX/NL- and DISC1-induced signaling might be interconnected. Here, we show that NL1 binds to Kal-7, and alterations in NX-NL interactions lead to disturbances in the DISC1/Kal-7/Racl signalosome, thus allowing the activation of Racl.

\section{Materials and Methods}

2.1. Materials. The recombinant ectodomain of human neurexin 1 beta Fc Chimera (ecto-NX1 $\beta$ ) was obtained from R\&D Systems (catalog number 5268-NX-050; Abingdon, UK). An expression vector that encodes NL1-AB, pCAGNL1$\mathrm{AB}$ was a gift from Peter Scheiffele (Addgene plasmid \#15262) [13]. An expression vector that encodes Kal-7, pEAK10-HisMyc-Kal7 was a gift from Betty Eipper (Addgene plasmid \#25454) [14]. An expression vector that encodes mouse FLAG-myc-tagged DISC1 was purchased from Origene (Rockville, MD, USA). The PKA inhibitor H89 was obtained from Invivogen (Toulouse, France). The following antibodies were used in this study: rabbit anti-S897-NR1, anti-S896NR1 (1:500; Millipore, Billerica, MA, USA), phospho-Erk1/2, phospho-Akt (1:1000; Cell Signaling, Beverly, MA, USA), mouse anti- $\beta$-tubulin (1:50,000; Sigma-Aldrich, St. Louis, MO, USA), anti-HA tag (Abcam, Cambridge, UK), anti-myctag (Sigma-Aldrich), anti-Kal-7 (Abcam), and IRDye secondary antibodies (1:20,000; Odyssey, Lincoln, NE, USA).

2.2. Cell Cultures. Cortical neurons were obtained on embryonic day 18 from Wistar rat embryos (Charles River Laboratories, Kisslegg, Germany) as described previously [15]. For each single experiment, the cortices from all embryos were dissected, cleared from blood vessels and meninges, pooled together, mechanically chopped, and trypsinized. The cells were then washed in the presence of DNase and trypsin inhibitor, resuspended, and seeded at a density of $2 \times 10^{5}$ cells $/ \mathrm{cm}^{2}$ in poly-D-lysine coated $(1 \mu \mathrm{g} / \mathrm{mL}) 6 \mathrm{~cm}$ Petri dishes (Nunc, Roskilde, Denmark) in Neurobasal medium supplemented with 2\% (v/v) B27, 1\% (v/v) GlutaMax, $100 \mathrm{U} / \mathrm{mL}$ penicillin, $100 \mu \mathrm{g} / \mathrm{mL}$ streptomycin, and $2 \%(\mathrm{v} / \mathrm{v})$ of $1 \mathrm{M}$ HEPES (all from Gibco, Waltham, MA, USA). At 3 DIV, cytosine $\beta$-D-arabinofuranoside (Ara-C) in concentration $2 \mu \mathrm{M}$ was added to inhibit the growth of nonneuronal cells. Cortical neurons were grown for 8 days. At this developmental stage the majority of synapses are immature. The advantage of using these young cultures is the high survival rate of neurons and general low background due to low level of spontaneous activity [16].

Human embryonic kidney 293 (HEK293) cells were obtained from the European Cell Culture Collection (Salisbury, UK) and maintained in Dulbecco's Modified Eagle's Medium (DMEM) supplemented with $10 \%$ (v/v) fetal calf serum, $2 \mathrm{~mm}$ GlutaMax, 100 units/mL penicillin, and $100 \mu \mathrm{g} / \mathrm{mL}$ streptomycin. All of the cell cultures were kept in a humidified incubator at $37^{\circ} \mathrm{C}$ in a $5 \% \mathrm{CO}_{2}$ atmosphere.

2.3. Immunoblotting. Cortical neurons were grown for 8 days before treatment. Thereafter, neurons were stimulated with
ecto-NX1 $\beta(0.01,0.05$, and $0.2 \mathrm{nM})$ for $5 \mathrm{~min}$. The cells were lysed, and proteins were separated by sodium dodecyl sulfatepolyacrylamide gel electrophoresis (SDS-PAGE) followed by immunoblotting using the antibody of interest. The bands were visualized using the Odyssey CLX Infrared Imaging System (Odyssey).

2.4. Rac1-GTP Assay. Cortical neurons were grown for 8 days and stimulated with ecto- $\mathrm{NX} 1 \beta(0.01,0.05$, and $0.2 \mathrm{nM})$ for $20 \mathrm{~min}$. The activation of Racl was measured with the Racl activity assay kit (Thermo Scientific, Rockford, IL, USA) according to the manufacturer's instructions. A fusion protein that consisted of the p21-binding domain of Pak1, which specifically binds to the active form of Racl (RacGTP) but not to the inactive form of Racl (Rac-GDP), was used for Rac-GTP precipitation. Immunoprecipitated samples were analyzed by SDS-PAGE and immunoblotting using a primary mouse anti-Racl antibody (1:1000; Thermo Scientific) and goat anti-mouse RDye secondary antibodies (1:20,000; Odyssey).

2.5. Real-Time Quantitative Polymerase Chain Reaction. Cortical neurons were grown for 8 days and treated with ecto$\mathrm{NX1} \beta$ (0.01, 0.05, and $0.2 \mathrm{nM}$ ) for $2 \mathrm{~h}$ (c-fos), $24 \mathrm{~h}$ (DISC1), or $48 \mathrm{~h}$ (NR1 splice variants). Total RNA was prepared using the GeneJET RNA Purification Kit (Thermo Scientific) according to the manufacturer's instructions. RNA integrity and quantity were verified using an Agilent 2100 Bioanalyzer (Agilent Technologies). Only samples with $\mathrm{RIN} \geq 8$ were used for further analyses. The RNA samples were stored at $-80^{\circ} \mathrm{C}$ until use. Quantitative reverse-transcription polymerase chain reaction (PCR) and the data analyses were performed as described previously [17] using the primers that are shown in Table 1 . All of the samples were run in duplicate. The relative levels of the PCR products in all of the samples were evaluated by the Pfaffl method [18].

2.6. Coimmunoprecipitation. HEK293 cells were transfected with cDNA expression vectors that encoded the proteins of interest. Forty-eight hours after transfection, cells were lysed in immunoprecipitation (IP) buffer (phosphate-buffered saline $[\mathrm{PBS}]$ and $1 \%$ NP40) supplemented with complete protease inhibitors (Roche), and cleared lysates were incubated with appropriate antibodies overnight. Immunoprecipitates were collected with Pierce protein A/G magnetic beads (Thermo Scientific), washed twice in IP buffer (once with PBS), and analyzed by SDS-PAGE and immunoblotting.

2.7. Data Analysis. The statistical analysis was performed using one-way analysis of variance (ANOVA) followed by Dunnett's multiple-comparison post hoc test, Sidak's multiple-comparison post hoc test, or one-sample $t$-test when appropriate using Prism 6 software (GraphPad, San Diego, CA, USA). 
TABLE 1: List of primers used in the study.

\begin{tabular}{|c|c|c|c|c|}
\hline \multirow{2}{*}{ Gene } & \multirow{2}{*}{ Accession number } & \multicolumn{2}{|c|}{ Primer } & \multirow{2}{*}{$\begin{array}{l}\text { Fragmen } \\
\text { size (bp) }\end{array}$} \\
\hline & & Sense & Antisense & \\
\hline$\overline{c-f o s}$ & NM_022197.2 & $5^{\prime}$ GGA GGA GGG AGC TGA CAG ATA C $3^{\prime}$ & $5^{\prime}$ GGT CAT TGG GGA TCT TGC AGG $3^{\prime}$ & 159 \\
\hline Discl & NM_175596.2 & $5^{\prime}$ TGC ACT CTG GGG TTC ATT CCG 3' & $5^{\prime}$ CCA TTC GAC GCC GGT GAT $3^{\prime}$ & 190 \\
\hline NR1-N1 & L08228.1 & $5^{\prime}$ ATC ATC CTG CTG GTC AGC GAC G $3^{\prime}$ & $5^{\prime}$ GCA CCT TCT CTG CCT TGG GTC C $3^{\prime}$ & 160 \\
\hline$N R 1-C 1$ & L08228.1 & $5^{\prime}$ CGT GAA CGT GTG GAG GAA GAA C $3^{\prime}$ & $5^{\prime}$ CTA CGT CTC TTG AAG CTG GAG G $3^{\prime}$ & 121 \\
\hline NR1-C2 & L08228.1 & $5^{\prime}$ CTC AAC CTC TCA GAT CCC TC $3^{\prime}$ & $5^{\prime}$ GCA GCA GGA CTC ATC AGT GT $3^{\prime}$ & 102 \\
\hline NR1-pan & L08228.1 & $5^{\prime}$ TGT ACG TCA AGC CCA CAA TGA G $3^{\prime}$ & $5^{\prime}$ ACC AGG TGC ACC TCA TAG GTA $3^{\prime}$ & 211 \\
\hline GAPDH & NM_017008.3 & $5^{\prime}$ AGC TGG TCA TCA ACG GGA AAC C 3' & $5^{\prime}$ CCT TCT CCA TGG TGG TGA AGA C $3^{\prime}$ & 126 \\
\hline
\end{tabular}

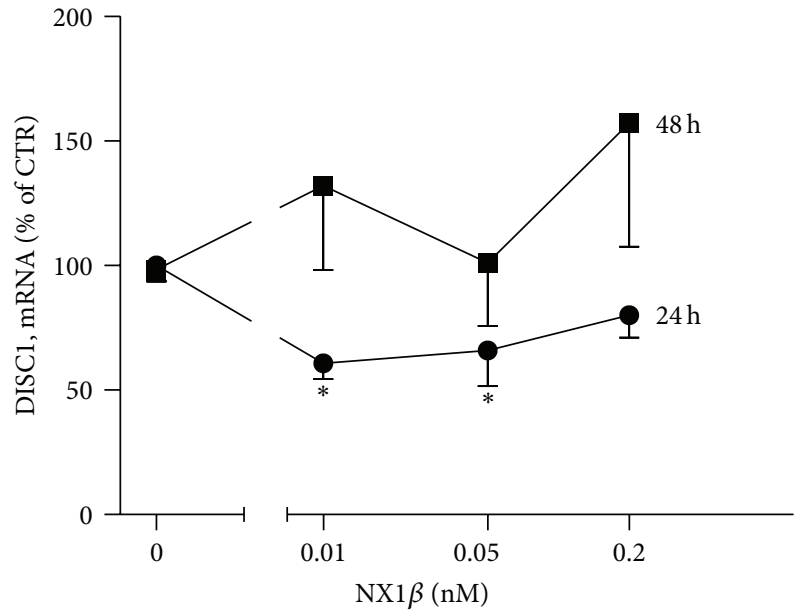

Figure 1: NX1 $\beta$ decreases the mRNA level of DISC1. Cortical neurons were grown for 8 days and subsequently treated with different concentrations of recombinant NX1 $\beta$ for (a) $24 \mathrm{~h}$ and (b) $48 \mathrm{~h}$. Total RNA was isolated, and mRNA levels were assessed using RT-PCR. The results from $n=3$ experiments are expressed as a percentage \pm SEM. ${ }^{*} P<0.05$, compared with the untreated control set at $100 \%$.

\section{Results and Discussion}

The expression of the NX1 gene appears to be regulated by a DISCl-dependent mechanism [7], and this regulation is important for brain development and function. We investigated whether this regulation might be reciprocal and whether NX might also affect the expression of Discl. Indeed, we found that $24 \mathrm{~h}$ treatment with ecto-NX1 $\beta$ decreased the mRNA level of Disc1 (Figure 1). This effect was inversely proportional to the dose of the protein. The highest concentration had no effect on Discl expression. Notably, the longer $48 \mathrm{~h}$ treatment with ecto-NX1 $\beta$ did not have a significant effect on Disc1 expression (Figure 1).

DISC1 has many identified protein interaction partners, including synaptic proteins [8]. We hypothesized that either NX or NL might interact with DISCl itself or might affect DISC1 interactions with other partners. Using LALIGN [18] to find the best local alignments between two sequences, we inspected the cytoplasmic domains of both NX1 (EAX00186.1) and NL1 (ADB12633.1) for homology to
DISC1 (Q9NRI5.3). We found that the cytoplasmic region of NL1 contains at least two sequence motifs that are homologous to DISC1 (Figure 2(a)). Moreover, these motifs are located in the DISC1 domain, which is crucial for binding with Kal-7 (amino acids 350-394 in DISC1) [10]. Hence, we examined whether NL1 might directly interact with Kal-7. The interaction was confirmed by co-IP from heterologous cells (Figure 2(b)). Moreover, in the presence of DISCl, the binding of Kal-7 to NL1 decreased substantially (Figure 2(c)), suggesting possible competition between DISC1 and NL1 for binding to Kal-7. In contrast, the presence of NL1 did not affect the DISC1-Kal-7 interaction, suggesting that DISC1 must first be released from Kal-7 to allow NL1-Kal-7 interactions.

The interaction between Kal-7 and DISC1 depends on synaptic activity. Upon neuronal activation, DISC1 dissociates from Kal-7, which in turn leads to the activation of Racl [10]. Neuronal activity also leads to the destabilization and shedding of both NX1 and NL1 from the synaptic membrane [3-6]. Additionally, treatment with recombinant ecto-NX1 $\beta$ mimics some of the effects of neuronal activity associated with the cleavage of NL1 from the postsynaptic membrane [6]. We observed that treatment with ecto-NX1 $\beta$ resulted in an increase in the mRNA level of the immediate early gene c-fos (Figure 3), indirectly supporting the hypothesis that treatment with soluble NX1 augments neuronal activity. Thus, we tested whether ecto-NX1 $\beta$ itself affects Racl activation. As shown in Figure 4, ecto-NX1 $\beta$ increased the level of RaclGTP at a concentration as low as $0.01 \mathrm{nM}$. At higher concentrations, ecto-NX1 $\beta$ did not have a statistically significant effect on Racl-GTP.

The signaling of Kal-7 to Racl is coupled with neuronal activity via NMDA receptors [10]. Therefore, we examined whether NX can modulate the phosphorylation levels of an obligatory subunit of the NMDA receptor, NR1. NR1 can be phosphorylated at three distinct serine residues (S897, S896, and S890) in the intracellular carboxyl tail region [19]. Active protein kinase C (PKC) phosphorylates the NR1 subunit at S890 and S896 [20], whereas the activation of cyclic adenosine monophosphate- (cAMP-) dependent protein kinase A (PKA) leads to phosphorylation at $\mathrm{S} 897$ [21]. As shown in Figure 5(a), treatment with ecto-NX1 $\beta$ led to phosphorylation at both S896 and S897. Interestingly, the level of Ser896 was markedly increased by the highest concentration of ecto-NX1 $\beta(0.2 \mathrm{nM})$, whereas the level of 


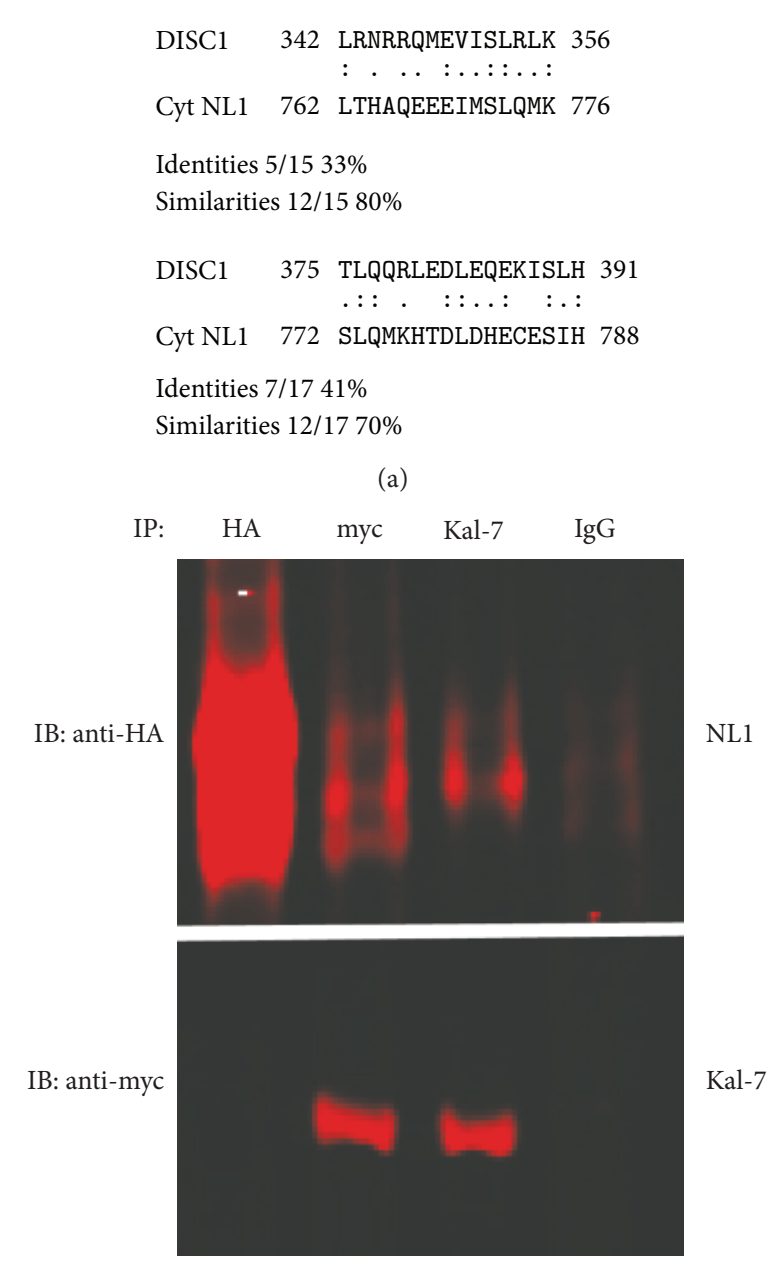

(b)

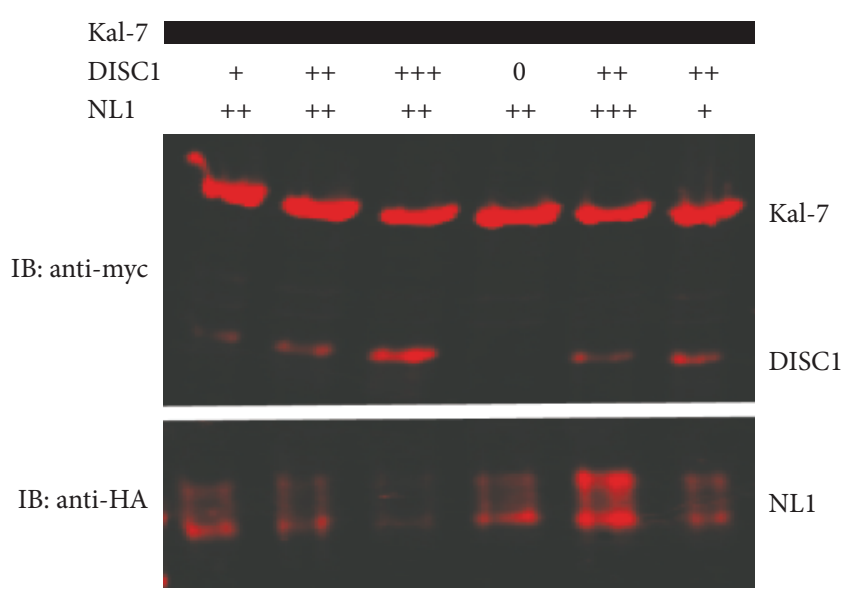

(c)

FIgURE 2: Neuroligin 1 interacts with kalirin 7. (a) $293 \mathrm{~T}$ cells were transfected with HA-tagged NL1-AB or myc-tagged Kal-7. Cell lysates were mixed and subsequently immunoprecipitated with anti$\mathrm{HA}$, anti-myc, or anti-Kal antibody, and immunoblots were probed with the indicated antibodies. Mouse IgG was used as a control. (b) HEK293 cells were transfected with HA-tagged NL1-AB or myctagged Kal-7 or FLAG-tagged DISC1. Cell lysates were mixed as indicated and subsequently immunoprecipitated. Immunoblots were probed with the indicated antibodies. Representative immunoblots of $n=3$ experiments are shown.

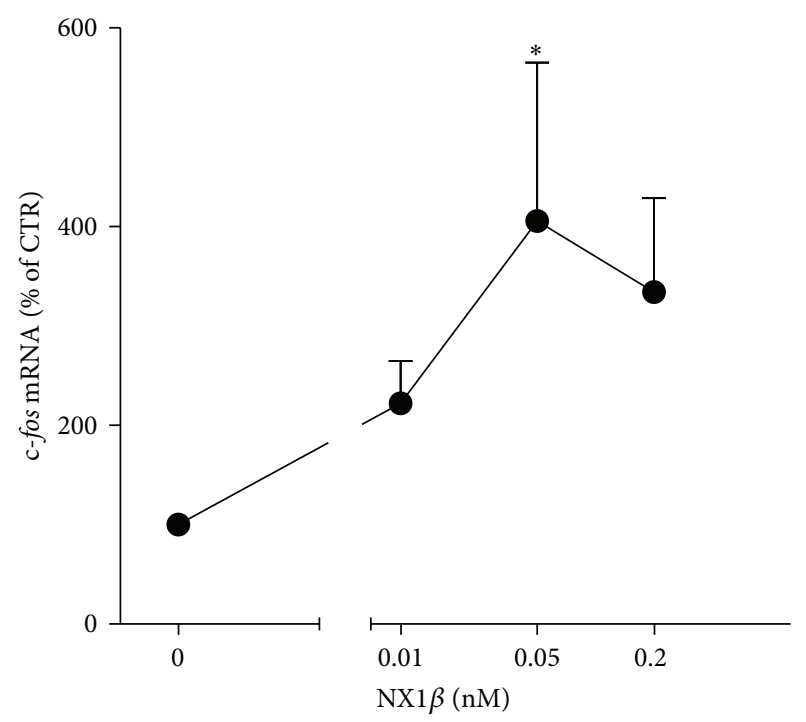

Figure 3: NX1 $\beta$ activates Racl. Cortical neurons were grown for 8 days and subsequently treated with different concentrations of recombinant $\mathrm{NX1} \beta$ for $20 \mathrm{~min}$, and the level of active Racl (RaclGTP) was analyzed using immunoblotting. The results from $n=4$ experiments are expressed as a percentage \pm SEM. ${ }^{*} P<0.05$, compared with the untreated control set at $100 \%$.

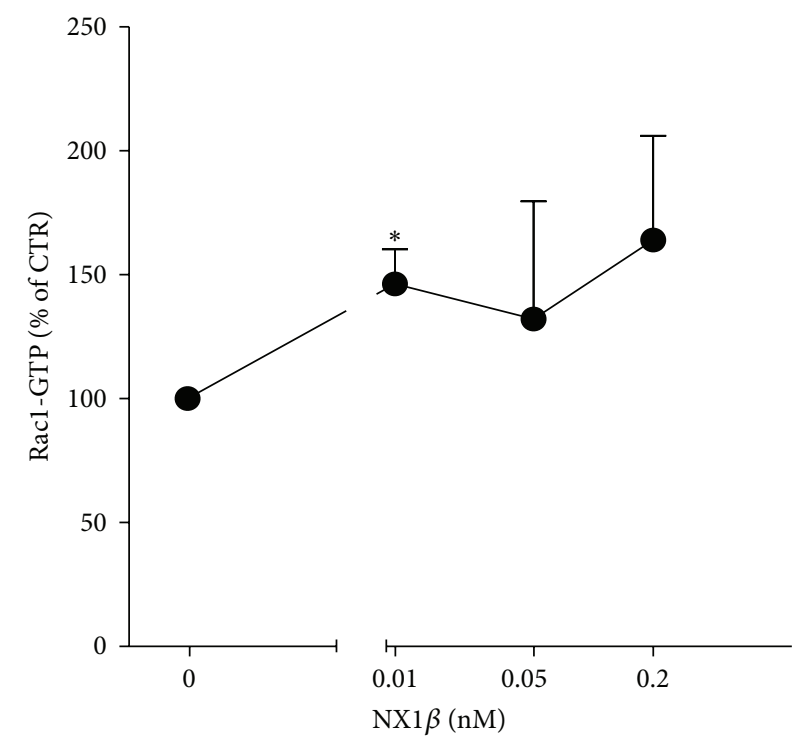

FIGURE 4: NX1 $\beta$ increases the mRNA level of the immediate early gene c-fos. Cortical neurons were grown for 8 days and treated with different concentrations of recombinant NRXN1b for $2 \mathrm{~h}$. Total RNA was isolated, and mRNA levels were assessed using RT-PCR. The results from $n=4$ experiments are expressed as a percentage \pm SEM. ${ }^{*} P<0.05$, compared with the untreated control set at $100 \%$.

Ser897 was significantly increased by $0.05 \mathrm{nM}$ ecto-NX1 $\beta$. In contrast to PKC, PKA responses are strong and brief. One of the targets that PKA phosphorylates and activates is phosphodiesterase, which rapidly lowers cAMP levels [22]. This might explain the differential effect of ecto- $\mathrm{NX1} \beta$ on PKA- and PKC-dependent phosphorylation sites in NR1. 


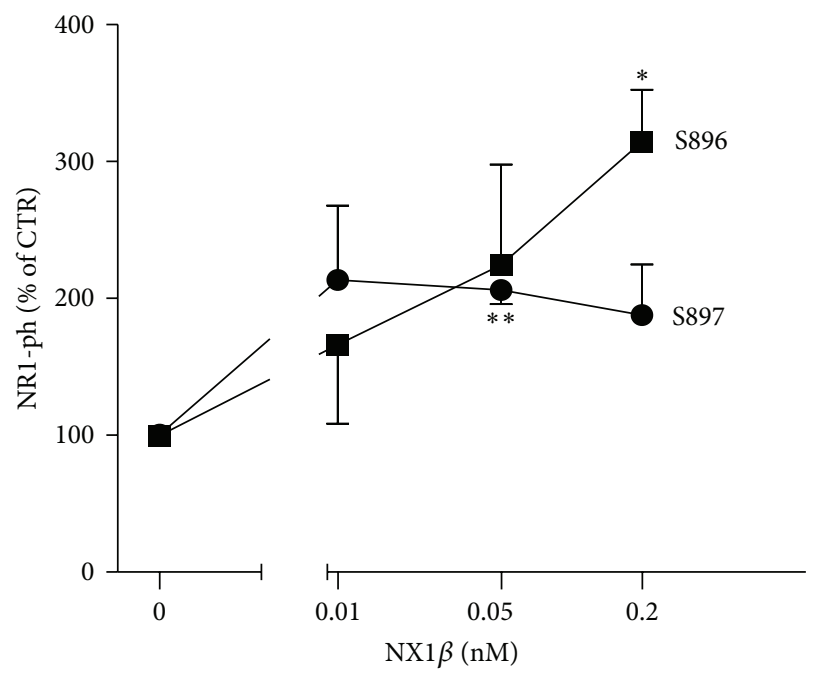

(a)

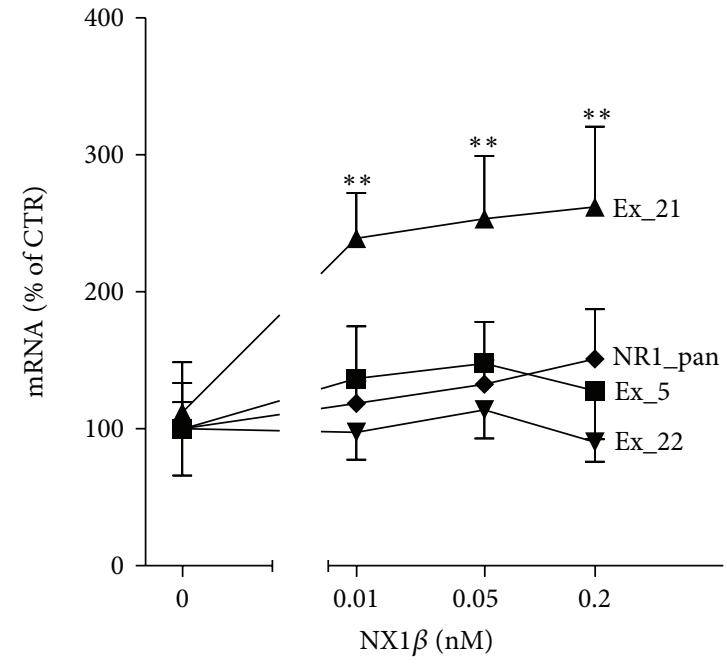

(b)

FIGURE 5: NX1 $\beta$ differentially affects the phosphorylation and expression of NR1. (a) Cortical neurons were grown for 8 days, subsequently treated with different concentrations of recombinant NX1 $\beta$ for $5 \mathrm{~min}$, and further immunoblotted for the detection of NR1 phosphorylation at S896 and S897. Representative immunoblots of three to four individual experiments are shown. The results from $n \geq 4$ experiments are expressed as a percentage \pm SEM. ${ }^{*} P<0.05$, compared with the untreated control set at $100 \%$. (b) Cortical neurons were grown for 8 days and treated with different concentrations of recombinant NX1 $\beta$ for $48 \mathrm{~h}$. Total RNA was isolated, and mRNA levels were assessed using RT-PCR. The results from $n=5$ experiments are expressed as a percentage \pm SEM. ${ }^{*} P<0.05$, compared with the untreated control set at $100 \%$.

NR1 exists in many isoforms through the alternative splicing of exons 5, 21, and 22. Exon 5, which encodes the extracellular N-terminal domain, is known to modulate the pharmacological properties of NMDA receptors [19]. Exon 21 and exon 22 (also known as the $\mathrm{C} 1$ and $\mathrm{C} 2$ cassettes, resp.) encode the intracellular C-terminal domain. mRNA splicing at these exons regulates protein-protein interactions, receptor trafficking, and NR1 phosphorylation [19]. We found that long-term $48 \mathrm{~h}$ treatment but not $24 \mathrm{~h}$ treatment (data not shown) with ecto-NX1 $\beta$ dose-dependently increased the relative expression levels of the NR1-C1 splice variant (Figure 5(b)) but not other splice variants.

The $\mathrm{Cl}$ cassette contains two independent endoplasmic reticulum (ER) retention motifs and plays a unique role in the trafficking of NR1 subunits to the cell surface. Thus, the effect of ecto-NX1 $\beta$ on NMDA receptor trafficking appears to be time-dependent because short-term treatment increased NR1 phosphorylation, which might promote the forward trafficking of NMDA receptors to the surface, but prolonged treatment led to the retention of NR1 in the ER.

Finally, we investigated which signaling pathways are activated by ecto-NX1 $\beta$ downstream of the NMDA receptor and Kal-7. Three kinases that represent three different signaling cascades, ERK1/2, p38 MAPK, and Akt (PKB), are known to mediate signal transduction that underlies neuronal activity in response to the activation of NMDA receptors $[23,24]$. As shown in Figure 6, ecto-NX1 $\beta$ had an effect on the phosphorylation levels of ERK1/2 (Figure 6(a)) and Akt (Figure 6(b)) but not p38 (Figure 6(c)). The effect on ERK1/2 and Akt was most pronounced at low concentrations and not seen at the highest concentration. To confirm the involvement of PKA in the NX-induced signaling pathway, we concomitantly treated cultured cortical neurons with ecto-NX1 $\beta$ and the specific PKA inhibitor H89. H89 abolished the effect of $10 \mu \mathrm{M}$ ecto-NX1 $\beta$ on ERK1/2 phosphorylation and had no effect in control (nonstimulated) cultures (Figure 6(d)). We did not observe any effect of the PKC inhibitor Calphostin C on NX-mediated ERK1/2 phosphorylation (data not shown).

Our data indicate that the NX1:NL1 complex is involved in the formation of the DISCl/Kal-7/Racl "signalosome," which is an important determinant of the neuronal activitydependent morphological and functional plasticity of dendritic spines [10]. The overexpression of either Kal-7 [14] or Racl led to an increase in spine size [10], and a similar effect was observed with DISCl downregulation [10]. The NX-NL complex is also involved in remodeling dendritic spines. Importantly, NL can act by itself to increase the number of synapses, but NX-NL binding is essential for the increase in apparent synapse size [25]. Except NX, NL1 also interacts extracellularly with NMDA receptor and this interaction is crucial for the synaptic recruitment and retention of NMDARs at glutamatergic synapses [26]. We suggest (see Figure 7) that, at the baseline condition, NL1 forms a homodimer [1] interacting with NMDA receptor while DISC1 binds Kal-7, blocking the access of Kal-7 to Racl. Upon synaptic activity and activation of NMDA receptors, NX1 $\beta$ is cleaved from the presynaptic membrane what leads to the partial cleavage of NL1 from the postsynaptic membrane followed by the release of NL1 from NMDA receptor and DISC1 from Kal-7. After the shedding, NL1 is no longer a dimer which probably changes the mobility of N-terminally truncated NL1, and it can diffuse allowing NL1 to bind Kal-7 and in turn lead to the activation of Racl. Additionally, binding of 


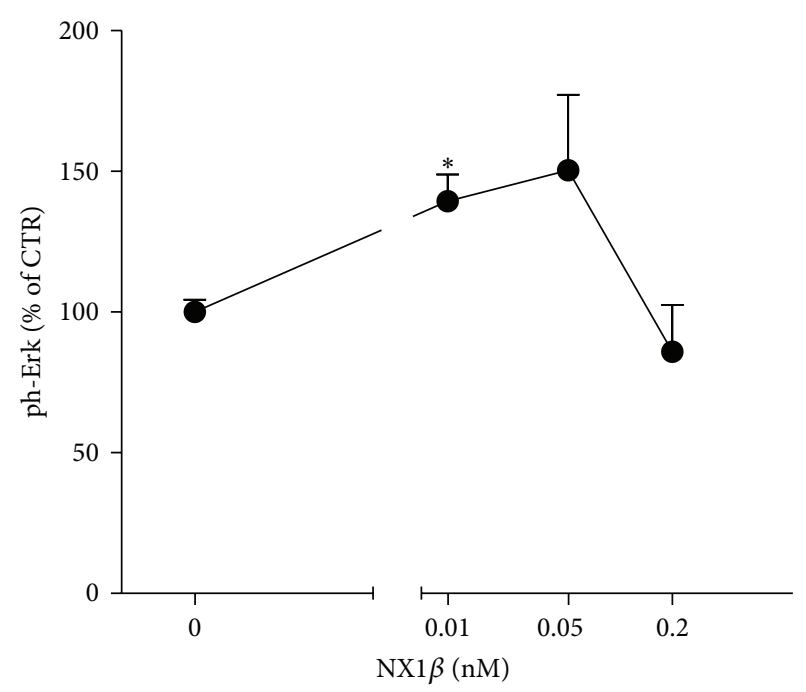

(a)

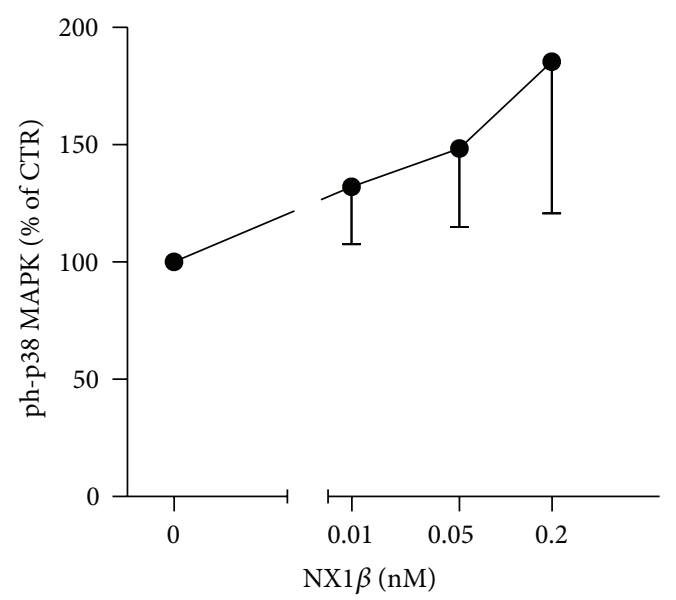

(c)

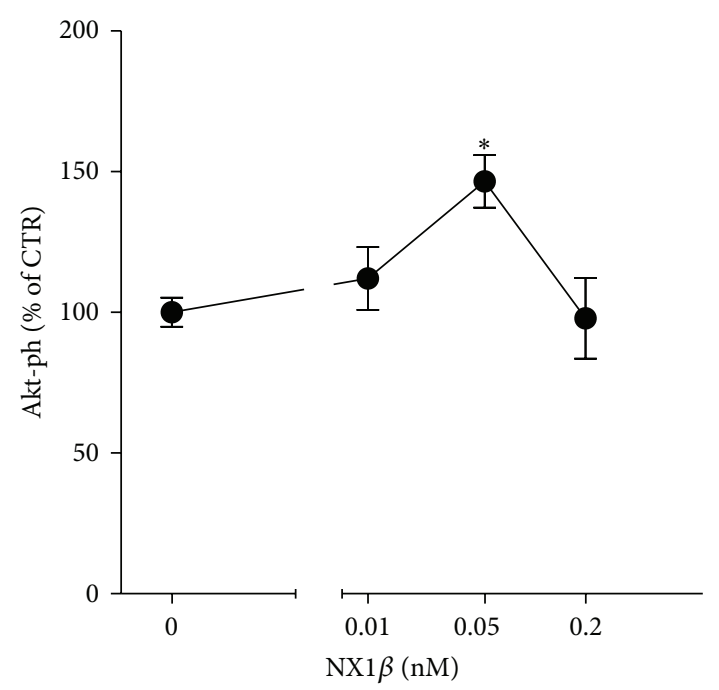

(b)

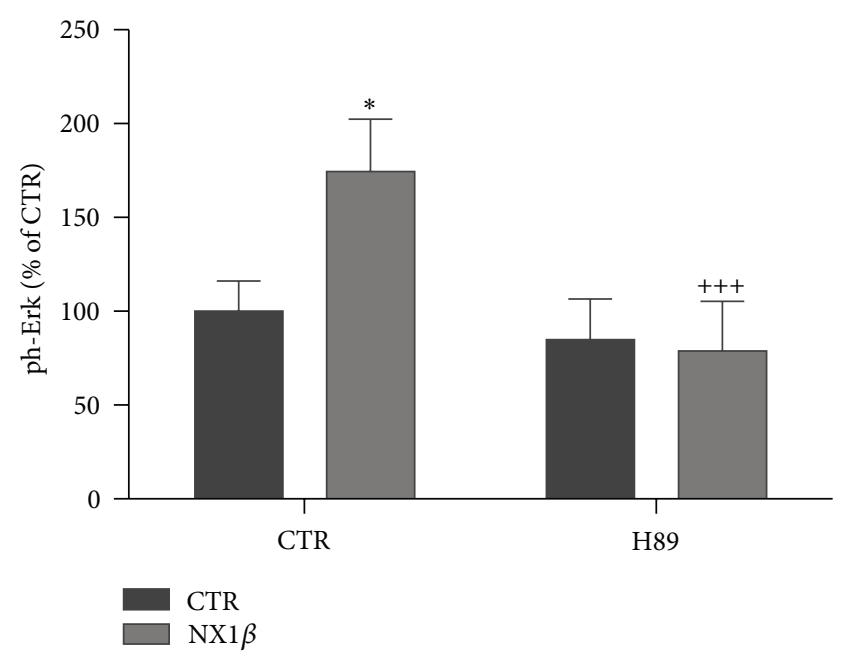

(d)

FIGURE 6: NX1 $\beta$ activates ERK1/2 and Akt kinases. Cortical neurons were grown for 8 days, subsequently treated with different concentrations of recombinant NX1 $\beta$ for $5 \mathrm{~min}$, and further immunoblotted for the detection of (a) ERK1/2, (b) p38 MAPK, and (c) Akt. (d) Effect of the PKA inhibitor H89 on NX1 $\beta$-induced ERK1/2 phosphorylation. The results from $n \geq 4$ experiments are expressed as a percentage \pm SEM. ${ }^{*} P<0.05$, compared with the untreated control set at $100 \%$.

ecto-NX1 $\beta$ to NL1 results in NR1 subunit phosphorylation at S896 and S897 and phosphorylation of ERK and Akt. Interestingly, the $24 \mathrm{~h}$ exposure to ecto-NX1 $\beta$ results in the diminished level of DISCl expression (Figure 1) and increased NL1 shedding from the cell surface [6] while prolonged (48 h) treatment with ecto-NX1 $\beta$ did not have any effect on mRNA level of DISC1 suggesting that all these events are reversible, in a sort of equilibrium, because the dynamics of these events determines spine plasticity.

Some mutations and structural variations in both the NX1 and DISC1 genes have been associated with schizophrenia and autism spectrum disorders [26-28]. The common denominator for these diseases is most likely disturbances in neuronal connectivity in the brain $[29,30]$. Interestingly, the abnormal expression of $N X 1$ in mice with mutated DISC1 is most prominent during the stages of synapse formation, neuronal maturation, and the general development of neuronal connectivity [7]. The DISC1/Kal-7/Racl signalosome also plays a role in neuronal development and may also contribute to the juvenile onset of mental diseases [31-33]. Our data support the functional connection between the DISC1/Kal-7/Racl signalosome and NX1 $\beta$-NL1 complex. This interaction might play an important role in establishing proper neural connectivity, and any disturbance of which might underlie pathologies seen in schizophrenia and autism spectrum disorders.

\section{Conclusion}

We found physical and functional connections between the NX1 $\beta$-NL1 complex and the DISC1/Kal-7/Racl signalosome. The NX1 $\beta$-NL1 complex appears to regulate the signalosome, 

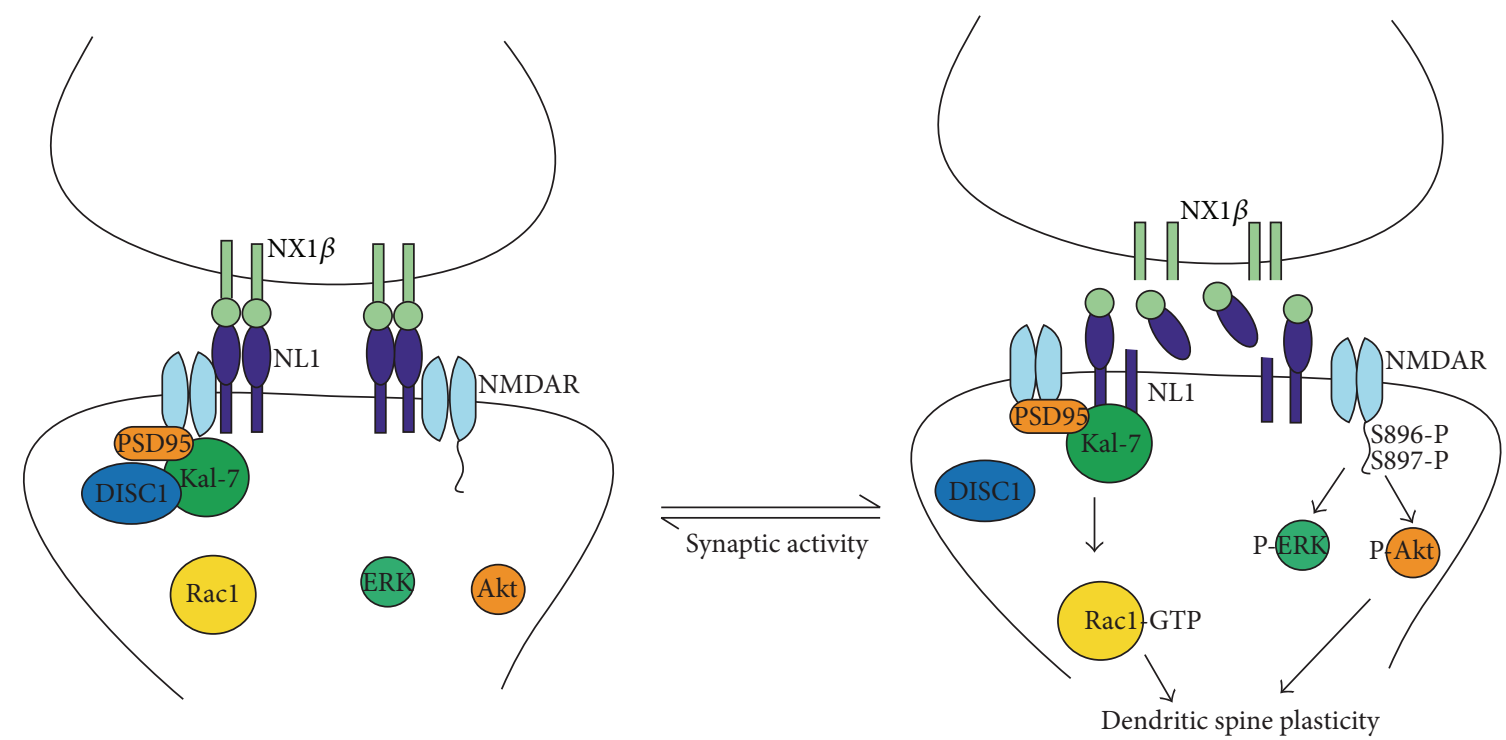

FIGURE 7: Schematic illustration of possible consequences of the NR1:NL1 interaction with the DISC1/Kal-7/Racl signalosome. At the baseline condition, NL1 forms homodimer interacting with NMDA receptor while DISC1 binds Kal-7, blocking the access of Kal-7 to Rac1. Upon synaptic activity and activation of NMDA receptors, NX1 $\beta$ is cleaved from the presynaptic membrane leading to partial cleavage of NL1 from the postsynaptic membrane followed by the release of NL1 from NMDA receptor and DISC1 from Kal-7. After the shedding, NL1 is no longer a dimer which probably changes the mobility of N-terminally truncated NL1, and it can diffuse allowing NL1 to bind Kal-7 and in turn lead to the activation of Racl. These events eventually allow NL1 to bind Kal-7 and in turn lead to the activation of Racl. Additionally, binding of ecto-NX1 $\beta$ to NL1 results in NR1 subunit phosphorylation at S896 and S897 and phosphorylation of ERK and Akt.

and this regulation is most likely closely coupled with synaptic activity and the activation of NMDA receptors.

\section{Conflict of Interests}

The authors declare that there is no conflict of interests regarding the publication of this paper.

\section{Acknowledgments}

The authors would like to thank the Lundbeck Foundation (R34-2009-3762 to Sylwia Owczarek) and Danish Research Councils for financial support. The funding sources had no involvement in the planning or preparation of this paper.

\section{References}

[1] M. L. Bang and S. Owczarek, "A matter of balance: Role of neurexin and neuroligin at the synapse," Neurochemical Research, vol. 38, no. 6, pp. 1174-1189, 2013.

[2] C. Kitamura, M. Takahashi, Y. Kondoh, H. Tashiro, and T. Tashiro, "Identification of synaptic activity-dependent genes by exposure of cultured cortical neurons to tetrodotoxin followed by its withdrawal," Journal of Neuroscience Research, vol. 85, no. 11, pp. 2385-2399, 2007.

[3] N. Bot, C. Schweizer, S. B. Halima, and P. C. Fraering, "Processing of the synaptic cell adhesion molecule neurexin- $3 \beta$ by Alzheimer disease $\alpha$ - and $\gamma$-secretases," Journal of Biological Chemistry, vol. 286, no. 4, pp. 2762-2773, 2011.

[4] R. T. Peixoto, P. A. Kunz, H. Kwon et al., "Transsynaptic signaling by activity-dependent cleavage of neuroligin-1," Neuron, vol. 76, no. 2, pp. 396-409, 2012.
[5] C. A. Saura, E. Servián-Morilla, and F. G. Scholl, "Presenilin $/ \gamma$ secretase regulates neurexin processing at synapses," PLoS ONE, vol. 6, no. 4, Article ID e19430, 2011.

[6] K. Suzuki, Y. Hayashi, S. Nakahara et al., "Activity-dependent proteolytic cleavage of neuroligin-1," Neuron, vol. 76, no. 2, pp. 410-422, 2012.

[7] S. M. Brown, S. J. Clapcote, J. K. Millar et al., "Synaptic modulators Nrxn1 and Nrxn3 are disregulated in a Discl mouse model of schizophrenia," Molecular Psychiatry, vol. 16, no. 6, pp. 585587, 2011.

[8] N. J. Bradshaw and D. J. Porteous, "DISC1-binding proteins in neural development, signalling and schizophrenia," Neuropharmacology, vol. 62, no. 3, pp. 1230-1241, 2012.

[9] F. H. F. Lee, M. P. Fadel, K. Preston-Maher et al., "Disc1 point mutations in mice affect development of the cerebral cortex," Journal of Neuroscience, vol. 31, no. 9, pp. 3197-3206, 2011.

[10] A. Hayashi-Takagi, M. Takaki, N. Graziane et al., "Disruptedin-Schizophrenia 1 (DISC1) regulates spines of the glutamate synapse via Racl," Nature Neuroscience, vol. 13, no. 3, pp. 327$332,2010$.

[11] J. K. Millar, S. Christie, and D. J. Porteous, "Yeast two-hybrid screens implicate DISC1 in brain development and function," Biochemical and Biophysical Research Communications, vol. 311, no. 4, pp. 1019-1025, 2003.

[12] Z. Xie, D. P. Srivastava, H. Photowala et al., "Kalirin-7 controls activity-dependent structural and functional plasticity of dendritic spines," Neuron, vol. 56, no. 4, pp. 640-656, 2007.

[13] B. Chih, L. Gollan, and P. Scheiffele, "Alternative splicing controls selective trans-synaptic interactions of the neuroliginneurexin complex," Neuron, vol. 51, no. 2, pp. 171-178, 2006.

[14] P. Penzes, R. C. Johnson, M. R. Alam, V. Kambampati, R. E. Mains, and B. A. Eipper, "An isoform of Kalirin, a brain-specific GDP/GTP exchange factor, is enriched in the postsynaptic 
density fraction," The Journal of Biological Chemistry, vol. 275, no. 9, pp. 6395-6403, 2000.

[15] D. K. Ditlevsen, S. Owczarek, V. Berezin, and E. Bock, "Relative role of upstream regulators of Akt, ERK and CREB in NCAMand FGF2-mediated signalling," Neurochemistry International, vol. 53, no. 5, pp. 137-147, 2008.

[16] Y.-C. Lin, Z.-H. Huang, I. S. Jan et al., "Development of excitatory synapses in cultured neurons dissociated from the cortices of rat embryos and rat pups at birth," Journal of Neuroscience Research, vol. 67, no. 4, pp. 484-493, 2002.

[17] T. Brudek, K. Winge, T. K. Agander, and B. Pakkenberg, "Screening of toll-like receptors expression in multiple system atrophy brains," Neurochemical Research, vol. 38, no. 6, pp. 12521259, 2013.

[18] M. W. Pfaffl, "A new mathematical model for relative quantification in real-time RT-PCR," Nucleic Acids Research, vol. 29, no. 9, article e45, 2001.

[19] B.-S. Chen and K. W. Roche, "Regulation of NMDA receptors by phosphorylation," Neuropharmacology, vol. 53, no. 3, pp. 362368, 2007.

[20] W. G. Tingley, M. D. Ehlers, K. Kameyama et al., "Characterization of protein kinase A and protein kinase $\mathrm{C}$ phosphorylation of the N-methyl-D-aspartate receptor NR1 subunit using phosphorylation site-specific antibodies," Journal of Biological Chemistry, vol. 272, no. 8, pp. 5157-5166, 1997.

[21] D. B. Scott, T. A. Blanpied, G. T. Swanson, C. Zhang, and M. D. Ehlers, "An NMDA receptor ER retention signal regulated by phosphorylation and alternative splicing," The Journal of Neuroscience, vol. 21, no. 9, pp. 3063-3072, 2001.

[22] G. Shaulsky, D. Fuller, and W. F. Loomis, "A cAMP-phosphodiesterase controls PKA-dependent differentiation," Development, vol. 125, no. 4, pp. 691-699, 1998.

[23] G. Sutton and L. J. Chandler, "Activity-dependent NMDA receptor-mediated activation of protein kinase B/Akt in cortical neuronal cultures," Journal of Neurochemistry, vol. 82, no. 5, pp. 1097-1105, 2002.

[24] W. E. Babiec, R. Guglietta, S. A. Jami, W. Morishita, R. C. Malenka, and T. J. O’Dell, "Ionotropic NMDA receptor signaling is required for the induction of long-term depression in the mouse hippocampal CA1 region," Journal of Neuroscience, vol. 34, no. 15, pp. 5285-5290, 2014.

[25] J. Ko, C. Zhang, D. Arac, A. A. Boucard, A. T. Brunger, and T. C. Südhof, "Neuroligin-1 performs neurexin-dependent and neurexin-independent functions in synapse validation," The EMBO Journal, vol. 28, no. 20, pp. 3244-3255, 2009.

[26] E. C. Budreck, O.-B. Kwon, J. H. Jung et al., "Neuroligin-1 controls synaptic abundance of NMDA-type glutamate receptors through extracellular coupling," Proceedings of the National Academy of Sciences of the United States of America, vol. 110, no. 2, pp. 725-730, 2013.

[27] A. N. Voineskos, T. A. P. Lett, J. P. Lerch et al., "Neurexin1 and frontal lobe white matter: an overlapping intermediate phenotype for schizophrenia and autism spectrum disorders," PLoS ONE, vol. 6, no. 6, Article ID e20982, 2011.

[28] D. St Clair, D. Blackwood, W. Muir et al., "Association within a family of a balanced autosomal translocation with major mental illness," The Lancet, vol. 336, no. 8706, pp. 13-16, 1990.

[29] J. K. Millar, J. C. Wilson-Annan, S. Anderson et al., "Disruption of two novel genes by a translocation co-segregating with schizophrenia," Human Molecular Genetics, vol. 9, no. 9, pp. 1415-1423, 2000.
[30] N. de Lacy and B. H. King, "Revisiting the relationship between autism and schizophrenia: toward an integrated neurobiology," Annual Review of Clinical Psychology, vol. 9, pp. 555-587, 2013.

[31] G. M. G. Shepherd, "Corticostriatal connectivity and its role in disease," Nature Reviews Neuroscience, vol. 14, no. 4, pp. 278-291, 2013.

[32] A. Hayashi-Takagi, Y. Araki, M. Nakamura et al., "PAKs inhibitors ameliorate schizophrenia-associated dendritic spine deterioration in vitro and in vivo during late adolescence," Proceedings of the National Academy of Sciences of the United States of America, vol. 111, no. 17, pp. 6461-6466, 2014.

[33] P. Mandela and X.-M. Ma, "Kalirin, a key player in synapse formation, is implicated in human diseases," Neural Plasticity, vol. 2012, Article ID 728161, 9 pages, 2012. 

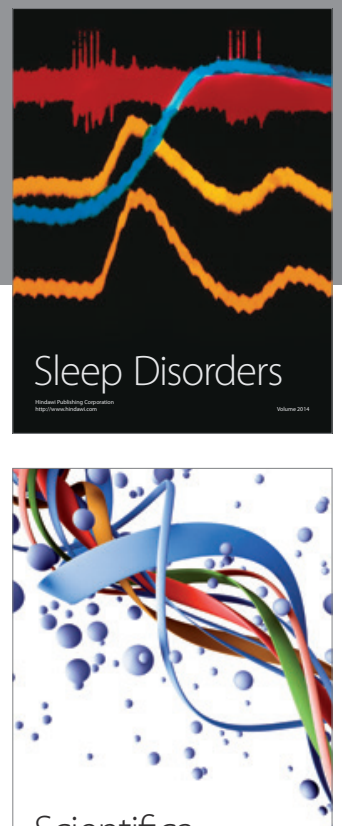

Scientifica
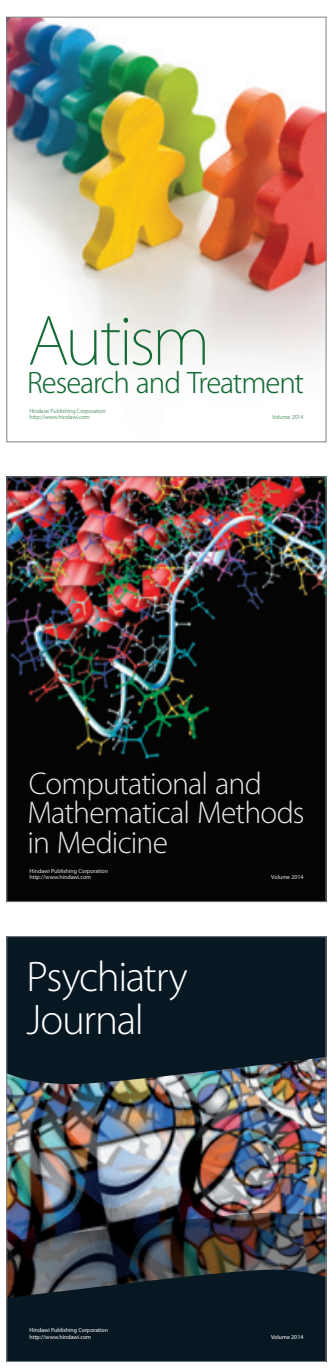
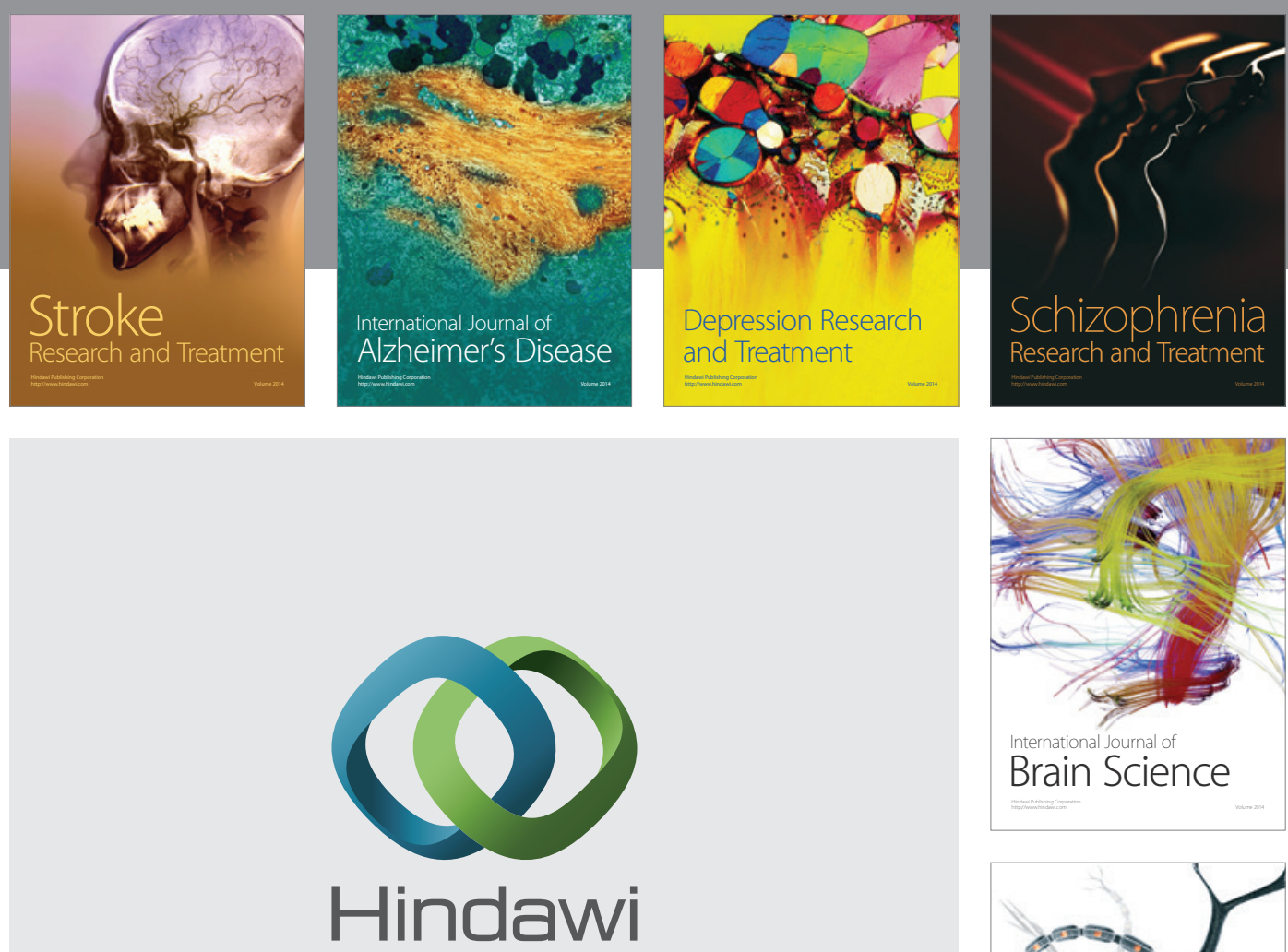

Submit your manuscripts at

http://www.hindawi.com
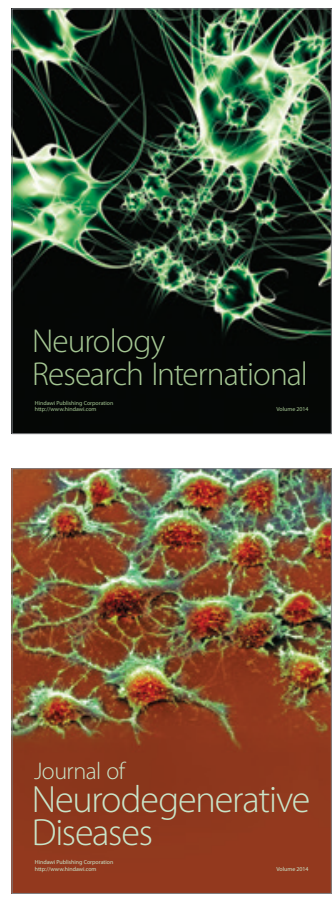

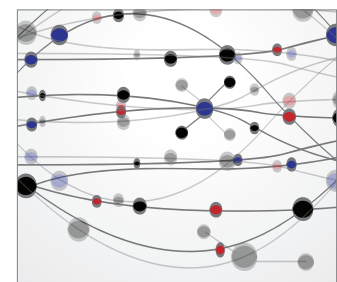

The Scientific World Journal
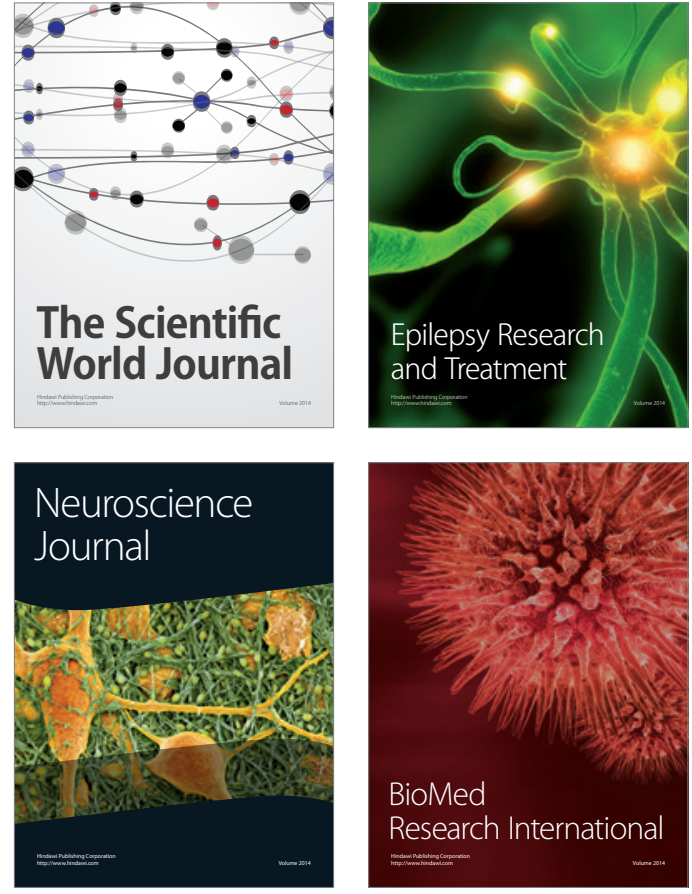

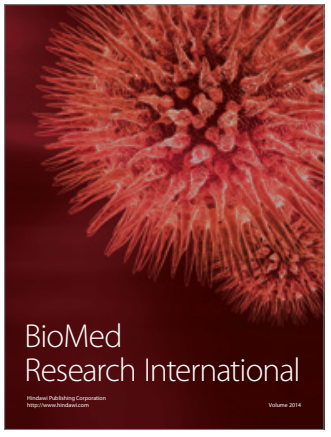

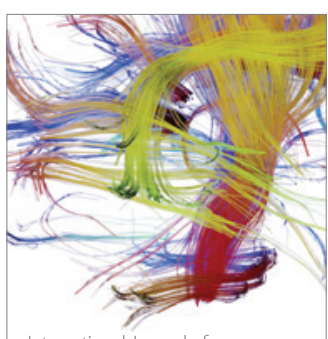

Brain Science

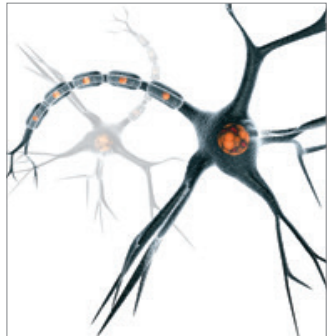

Neural Plasticity
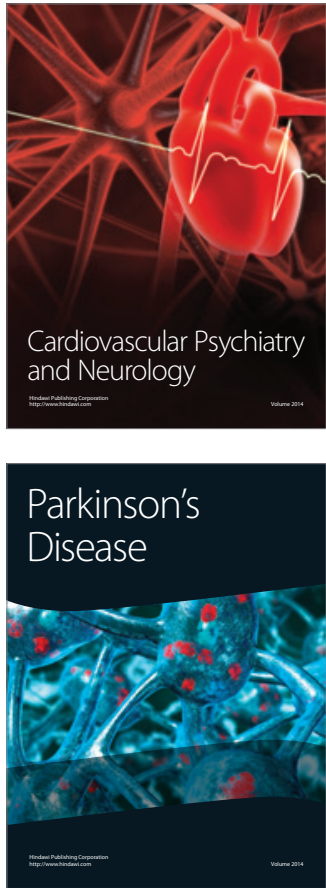